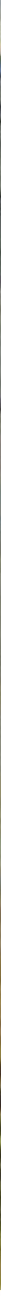

\title{
Rekonstruktion des Bauablaufs der ersten Bauphase des gotischen Meißner Doms
}

\author{
Günter Donath
}

Darstellung einer Kirchenbaustelle, u. a. mit einer Bauhütte, Werksteintransport und Mörtelmachen sowie das Vermauern. Die Mauerkronen sind mittels Mist zur Sicherung gegen Witterungseinflüsse abgedeckt aus: L'Histoire de Charles Martel, 1448/65, Brüssel, (Bibl. Royale, Ms. 6, fol. 554)
Die intensive wissenschaftliche Auseinandersetzung mit der mittelalterlichen Bauplanung und den Bauprozessen an den drei Chorbauten des Naumburger Doms, der Zisterzienser-Klosterkirche St.Marien in Schulpforte und des Meißner Doms im Zusammenhang mit der für die Landessaustellung „Der Naumburger Meister“ in Naumburg durchgeführten Bauforschung 2009/10 durch den Verfasser gab Veranlassung, wiederholt die Frage nach Baubeginn, Bauverlauf und Bauzeit der Ostteile des Meißner Doms zu stellen. Während Ernst Schubert ${ }^{1}$ auf Grund stilkritischer Vergleiche einen „Baubeginn vor 1260“ ausschloss, geht Matthias Donath ${ }^{2}$ bei seiner Datierung von einem Baubeginn „um 1250“ aus. Heinrich Magirius ${ }^{3}$ vermeidet gänzlich die Nennung konkreter Jahreszahlen; er ordnet „nach alter Überlieferung“ den Baubeginn der Amtszeit Bischofs Konrad (12401258) und die Vollendung des ersten großen Bauabschnittes Bischof Withego (1266-1293) zu. Al- lerdings hat sich durch jüngste Forschungen zum Naumburger Dom von Holger Kunde ${ }^{4}$ mit der Datierung der Fertigstellung des Naumburger Westchores „um 1249“ ein neuer Ansatzpunkt ergeben. Für Schulpforta ist der Baubeginn mit „1251“ durch einen Inschriftenstein am südöstlichen Polygonpfeiler und die Weihe 1268 urkundlich belegt. Damit ist für den vergleichsweise kleinen Bau immerhin eine Bauzeit von 17 Jahren anzunehmen. Bisher ist die Kunstgeschichtsforschung stets davon ausgegangen, dass die Bauleute von der Pförtner Chorbaustelle weiter nach Meißen gezogen sind und damit stilprägende Elemente weitergetragen haben.

Ein Vergleich der Steinmetzzeichen der drei Chorbauten sowie die der zusätzlich einbezogenen Templerkapelle in Iben ${ }^{5}$ kommt aber zu einem anderen Ergebnis ${ }^{6}$ : So erbringt ein Abgleich der Steinmetzzeichen des Naumburger Westchors mit denen des Meißner Doms und der Templerkapelle 
Iben das überraschende Resultat, dass von den 48 in Naumburg vorkommenden Steinmetzzeichenarten 18 in Meißen in der gleichen Form nachgewiesen werden können. Der Grad der Übereinstimmung tritt noch deutlicher hervor, wenn man die Anzahl der Steinmetzzeichen pro Steinmetzzeichenart berücksichtigt. Die 18 übereinstimmenden Steinmetzzeichen machen 64,2 Prozent des Steinmetzzeichenbestands des Naumburger Westchors aus. Von den 15 häufigsten Steinmetzzeichen sind elf in Meißen belegt, einige davon in sehr hohen Stückzahlen. Eine zufällige Übereinstimmung ist auch deshalb ausgeschlossen, weil sich unter den identischen Steinmetzzeichen solche befinden, die eine einzigartige, individuelle Gestalt haben, etwa das Herz oder der Hammer. Im Ergebnis lässt sich feststellen, dass 18 Steinmetzen, die im Naumburger Westchor tätig waren, auch auf der Meißner Baustelle gearbeitet haben. Erstaunlich ist, wie gering die Übereinstimmung in Schulpforta mit den Steinmetzzeichen des Naumburger Westchors ausfällt. Nur zwei Steinmetzzeichen kommen sowohl in Naumburg als auch in Schulpforta vor. Mit drei Belegen machen sie lediglich 4,6 Prozent des Steinmetzzeichenbestands in Schulpforta aus. Damit trifft die immer wieder vorgebrachte Vermutung, der Naumburger Westchor und der Chor der Klosterkirche Schulpforta seien von der gleichen Bauhütte errichtet worden, nicht zu. Vielmehr ergibt der Vergleich der Steinmetzzeichen, dass in Schulpforta eine eigenständige Gruppe von Steinmetzen tätig war, die nur partiell Berührungen mit Iben, Naumburg und Meißen hatte. Die identischen Steinmetzzeichen deuten auf eine Arbeitskräftewanderung hin, die sich von Iben über Naumburg nach Meißen vollzog. Von den Steinmetzen, die in Iben arbeiteten, zog ein Teil nach Naumburg, wo zusätzliche Kräfte hinzutraten. Die Naumburger Gruppe wiederum wanderte teilweise nach Meißen, wo sie sich abermals um neue Kräfte ergänzte. So sind von den elf Steinmetzzeichenarten, die in den unteren, ältesten Teilen des Meißner Domchors vorkommen, nur vier mit Naumburg identisch. Eine andere Wanderungsrichtung, etwa von Meißen über Naumburg nach Iben, ist aufgrund der künstlerischen Entwicklung, die in den Bauten $\mathrm{zu}$ erkennen ist, auszuschließen. Der Pförtner Chorbau ordnet sich nicht in diese Reihe ein, sondern wurde von eigenen Kräften errichtet. Dass schon aufgrund der geographischen Nähe von Meißen, Naumburg und Schulpforta - einzelne Steinmetzen zwischen den Baustellen hin- und hergewandert sind, ist plausibel. So können Steinmetzen aus Schulpforta zur Meißner Dombaustelle gewechselt sein und umgekehrt Steinmetzen aus Meißen in Schulpforta gearbeitet haben. Mit dieser für alle Beteiligten überraschenden Feststellung und „1249“ als ein neuer terminus ante quem für Meißen war also die Beantwortung der Frage nach der für die Ostteile des Meißner Doms anzunehmenden Bauzeit noch dringlicher geworden ${ }^{7}$.

\section{Bauherrschaft und Bauorganisation}

Bischof Konrad I. (1240-1258) erwirkte 1249/50 von der päpstlichen Kanzlei drei Ablässe. Die Urkunden erwähnen dort das Baugeschehen in Meißen zwar nicht ausdrücklich, doch ist es sehr wahrscheinlich, sie auf die damals angelaufenen Baumaßnahmen zu beziehen, die viel Geld erforderten. Der Bischof musste sich die Bauherrschaft mit dem Domkapitel teilen, das im 13. Jahrhundert zu einer rechtlich eigenständigen Einrichtung geworden war, die Urkunden ausstellte, Vermögenswerte verwaltete und den Bischof wählte. Die Bauverwaltung und -finanzierung wurde einer fabrica (Stiftsbaumeisterei) übertragen, die zwar erst 1271 urkundlich bezeugt ist, jedoch schon früher bestanden haben muss. Die Fabrica unterstand direkt dem Domkapitel. 1290 nahm der Domherr Konrad von Boritz als thesaurarius deren Aufsicht wahr. Die Leitung hatte ein magister fabricae inne, der nicht geistlichen Standes war. Ein solcher ist in Meißen zwar erst 1324 nachgewiesen, doch muss es in Anbetracht der Größe der seinerzeit anstehenden Bauaufgabe dieses Amt auch schon vorher gegeben haben.

Die auf der Baustelle tätigen Bau- und Werkmeister, Steinmetze und Bauhandwerker sind in den überlieferten Urkunden, die überwiegend finanzielle Sachverhalte regeln, weder namentlich noch durch Amtsbezeichnungen überliefert. Ihre Arbeit kann allein durch Baubefunde und den Vergleich mit der Struktur und Größe anderer Bauhütten dieser Zeit nachvollzogen werden. Dazu kann man Angaben zu Zahlen der Handwerker ebenso wie das Verhältnis der einzelnen Berufsgruppen zueinander in Erfahrungsberichten der Hütten in Straßburg, Regensburg und Köln heranziehen, die vom Umfang her ähnlich große und technisch anspruchsvolle Bauaufgaben zu erfüllen hatten, wie sie in Meißen mit dem ambitionierten Domneubau zur Mitte des 13. Jahrhunderts bestanden. Wie es bei diesen drei Hütten der Fall war, ist deshalb auch in Meißen eine Anzahl von Arbeitskräften heranzuziehen, die für den Steintransport auf dem Lande und auch auf dem Wasser sorgten. Bei der Menge der zu transportierenden Steine dürften täglich zwei Schiffe unterwegs gewesen sein. Der Transport zu Lande wurde üblicherweise mit von den Steinbrucharbeitern der jeweiligen Brüche erledigt.

Für den Meißner Dombau waren die Sandsteinbrüche in Grillenburg und Niederschöna ${ }^{8}$ bzw. im Lohmgrund und der Alten Poste ${ }^{9}$ maßgebliche Steinlieferanten. Ein Teil der Steinbrucharbeiter brachte als Rohbossierer bereits dort die durch Hohlmachen gebrochenen rohen Sandsteinblöcke in handhabbare Steinquadergrößen. In Meißen mussten dann zusätzliche Fuhrknechte mit Pferdeund Ochsengespannen die mittels Lastkähnen angelieferten Steinquader vom Anlegeplatz am Elbufer - wo sicher auch ein Materiallagerplatz mit einer Ausspanne und einer Schmiede eingerichtet war - zur hoch über der Elbe gelegenen Baustelle transportieren. Die Anzahl der in der er-
1 Edgar Lehmann/Ernst Schubert: Der Meißner Dom. Beiträge zur Baugeschichte und Baugestalt bis zum Ende des 13. Jahrhunderts. Berlin 1969; Ernst Schubert: Der Westchor des Naumburger Domes, der Chor der Klosterkirche in Schulpforta und der Meißener Domchor. In: Friedrich Möbius/Ernst Schubert (Hrsg.): Architektur des Mittelalters. Funktion und Gestalt. Weimar 1983, 160-183, zuletzt Ernst Schubert: Rezension zu Matthias Donath, Die Baugeschichte des Doms zu Meißen. In: Kunstchronik 54 (2001), S. 258, 259.

2 Matthias Donath: Die Baugeschichte des Doms zu Meißen 1250-1400. Beucha 2000.

3 Heinrich Magirius (Hrsg.): Forschungen zur Bau- und Kunstgeschichte des Meißner Doms. Bd. 1. Das Portal an der Westturmfront. Halle 1999, Bd. 2. Architektur und Skulptur des Meißner Doms im 13. und 14. Jahrhundert. Weimar 2001.

4 Holger Kunde: Der Westchor des Naumburger Doms und die Marienstiftskirche. Kritische Überlegungen zur Forschung. In: Enno Bünz, (Hrsg.): Religiöse Bewegungen im Mittelalter. Festschrift für Matthias Werner zum 65. Geburtstag. Weimar 2007, S. 213-238.

5 Wolfgang Bickel: Templerkapelle Iben. Baukunst und Spiritualität im Orden der Armen Ritter Christi. Worms 2009, S. 48-53.

6 Günter Donath/Matthias Donath: Zeugnisse mittelalterlicher Bauplanungen und Bauprozesse an den Chorbauten von Naumburg, Schulpforta und Meißen. In: Hartmut Krohm/Holger Kunde (Hrsg.): Der Naumburger Meister - Bildhauer und Architekt im Europa der Kathedralen. Bd. 2. Petersberg 2011, S. 1275-1291.

7 Hartmut Krohm und Leonhard Helten ist für die Anregung $\mathrm{zu}$ danken, die Bauzeit des Meißner Doms in Analogie zu den von Dombaumeister Arnold Wolff für den Kölner Dom angestellten Untersuchungen zu ermitteln. Alle Berechnungen des vorliegenden Aufsatzes beziehen sich auf Arnold Wolff: Chronologie der ersten Bauzeit des Kölner Doms 1248-1277. Köln 1968. 

nath: Der Dom zu Meißen - die Geschichte, die Konstruktion und die Konsolidierung des Steinbaus. In: das münster 51 (1998), S. 194-211.

9 Donath (wie Anm. 2), S. 64-69. 10 Marc-Carel Schurr: Gotische Architektur im mittleren Europa 1220-1300, München 2007, S.136

Steinmetzzeichen des Meißner Doms in den Bauphasen la und lb aus: Donath 2000 (wie Anm. 2), S. $90-91$
8 Günter Donath/Matthias Do-

sten Bauphase Ia, dem Bau der Fundamente und Substruktionen, gefundenen elf Steinmetzzeichen und die der anschließenden Bauphase Ib bei der Errichtung des Aufgehenden - insgesamt 68 Zeichen - lassen den Schluss zu, dass zunächst eine, später dann mehrere Versetzerkolonnen tätig waren. 68 Steinmetze sind vergleichsweise zur Kölner Dombaustelle, bei der nur 20-24 Steinmetze angesetzt waren, ein sehr hoher Personalbesatz. Er lässt sich vielleicht damit erklären, dass nicht alle 68 Steinmetze über die gesamte Bauzeit hinweg gleichzeitig tätig waren, sondern zeitversetzt in mehreren Kampagnen nach Meißen kamen. Dies bestätigt auch die Verteilung der Steinmetzzeichen in dem betrachteten Bauabschnitt.

Eine arbeitsfähige Versetzerkolonne bestand aus drei bis vier Mann. Diese transportierten den fertig behauenen Stein von der Hütte auf den Bauplatz und vermauerten ihn dort fachgerecht. Für solche Arbeiten kamen nur ausgebildete Steinmetzen in Frage. Um eine Versetzerkolonne zu beschäf-

\section{Die Steinmetzzeichen des Meißner Domes - Übersicht Gruppe A, Untergruppe A1 - A5}

\begin{tabular}{|l|l|l|l|l|}
\hline Gruppe & Nummern & Vorkommen & Bauphase & Datierung \\
\hline A 1 & $\begin{array}{l}1,16,29,60, \\
162,210,259, \\
262-265\end{array}$ & $\begin{array}{l}\text { Hoher Chor, } \\
\text { untere Bereiche des } \\
\text { Stifterjochs und des } \\
\text { Chorpolygons }\end{array}$ & 1a & um 1250 \\
\hline
\end{tabular}

Steinmetzzeichen

(mit Angabe der Steinmetzzeichen-Nummer und der Häufigkeit in der jeweiligen Gruppe)

\begin{tabular}{|c|c|c|c|c|}
\hline A 2 & $\begin{array}{l}\text { 1-50, 54 58, } \\
60,64,71,72, \\
164,165, \\
169-171, \\
196,209,210, \\
216-218, \\
258,260,261\end{array}$ & $\begin{array}{l}\text { Hoher Chor; } \\
\text { Querhaus; } \\
\text { Osttürme; } \\
\text { Achteckbau; } \\
\text { basilikales Joch; } \\
\text { Pfeiler N1, S1, S2; } \\
\text { Einwölbung von Chor, } \\
\text { Querhaus und basilikalem } \\
\text { Joch }\end{array}$ & $1 \mathrm{~b}$ & $1250-1270$ \\
\hline
\end{tabular}

\section{Steinmetzzeichen}

(mit Angabe der Steinmetzzeichen-Nummer und der Häufigkeit in der jeweiligen Gruppe)

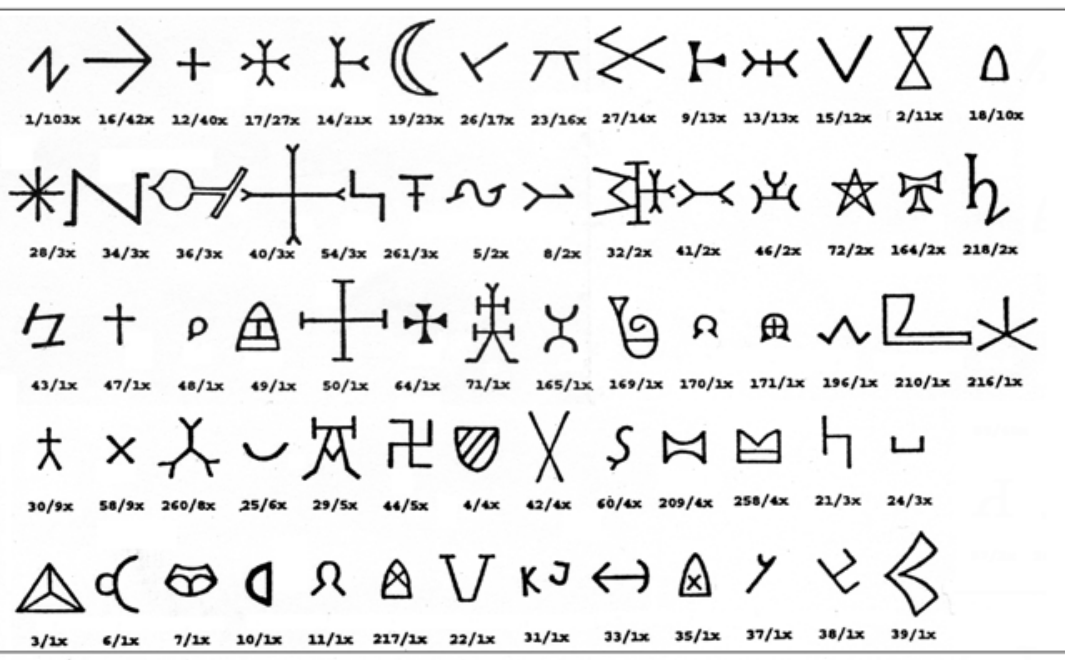

tigen, war in der Regel die drei- bis vierfache Anzahl Steinmetzen in der Hütte erforderlich, also 12 bis 16 Mann. Für den Bau der Fundamente müssen auch die dort beschäftigten Maurer, Schiffer, Steinbrecher, Mörtelmacher und Transportarbeiter (Haspelknechte) berücksichtigt werden. Hinzu kommen die Zimmerer, die für den Verbau der Fundamentgräben, später für den Bau der Arbeitsgerüste und der Schalgerüste zu sorgen hatten. Schließlich sind noch die Bauleitung und die Ausbildung des Nachwuchses zu bedenken. Neben dem Dombaumeister dürften mindestens noch ein Meisterknecht und ein Parlier oder wenigstens einer von beiden gestanden haben. Lehrlinge seien im Verhältnis 1:10 zu den Fachkräften angenommen.

Danach ergibt sich nebenstehende Zusammenstellung der am Dombau beschäftigten Personen. Dazu kommen noch von einem bestimmten Zeitpunkt an die Handwerker des Ausbaus, die ebenfalls der Hütte angehören: Vergolder, Maler, Glaser. Nimmt man noch die nicht der Hütte angehörenden Schiffsleute und Fuhrleute mit ihren Hilfsarbeitern für den Steintransport außerhalb der Baustelle hinzu, dann kommt man auf etwa 100-123 mit dem Dombau Beschäftigte. Diese Rechnung kann die Beschäftigtenzahl selbstverständlich nur annähernd genau erfassen. Völlig unklar ist dabei, wo diese Menschen alle gewohnt haben, wie sie verköstigt wurden und wo sich unter den beengten räumlichen Bedingungen auf dem Domberg überhaupt ein hinreichend großer Bauplatz mit den Hütten, Holz- und Steinlager und Kalksümpfen anlegen ließ.

\section{Bauplanung, Vermessung, Bauabsteckung und der Bau der Substruktionen}

Zwingende Voraussetzung für den Domneubau war, bestimmte Grundformen und -maße in Grund- und Aufriss festzulegen. Das Bild, welches das Domkapitel als Auftraggeber dabei für die Konzeption des gotischen Neubaus vor Augen hatte, sollte die „Erinnerungsgestalt“10 des zu diesem Zeitpunkt noch aufrecht stehenden romanischen Doms bewahren: ein dreischiffiges Langhaus, eine Chorapsis mit Flankentürmen und ausladendem Querhaus sowie der Doppelturmfront im Westen waren die charakteristischen Merkmale, die nun in modernste Bauformen gleichsam „übersetzt“ werden mussten.

Als Inspirationsquelle für die Durchbildung des Chores, mit einem im Sinne eines „Glashauses“ völlig aufgelösten 5/8-Schluss diente die dem Bauherren und Baumeister offenbar gleichermaßen bekannte Chorscheitelkapelle der Kathedrale in Amiens, während für die innere Durchbildung die durch den Naumburger Meister und seine Hütte entwickelte Formenwelt herangezogen wurde. Die neue Domkirche sollte als steinsichtiger Quaderbau konstruiert werden. Diese allgemeine Vorstellung von Bautyp und Dimension im Geiste des Baumeisters diente nun als Grundlage 
Dombaumeister

Meisterknecht, Parlier

Bildhauer (z. B. Zeichen „T“ an Lettner, Dorsale und Gewölbe)

Steinmetzen in der Hütte ( $68-11=57$, die aber nicht gleichzeitig anwesend waren)

Versetzer

Lehrlinge

Zimmerleute

Steinbrecher

Rohbossierer

Mörtelmacher, Schmiede, Haspelknechte

am Aufgehenden beschäftigte Personen

Fundamentmaurer

Steinmetzen in der Hütte

Lehrlinge

Steinbrecher Bruchstein

Zimmerer

Haspelknechte

an den Substruktionen beschäftigte Personen

insgesamt in der Dombauhütte beschäftigte Personen
11 Generell verlief - entgegen der immer wieder vehement vorgetragenen falschen Meinung - die Bauausführung bis zur Mitte des 13. Jahrhunderts weitgehend ohne vorher zeichnerisch festgelegte Projektierung.

12 Donath 2000 (wie Anm. 2) S. $50-58$ für die Bauausführung. ${ }^{11}$ Die architektonische Idee nahm erst in der empirischen Auseinandersetzung mit den örtlichen Gegebenheiten und dem Material im emporwachsenden Bau ihre endgültige Form an. Hier erst kam das baumeisterliche Wissen mit seinen Kenntnissen der Euklidschen Geometrie, des Satzes des Pythagoras oder Werken der Trigonometrie, die den Gebrauch von Sinus und Tangente zeigten, sowie der Algebra zur vollen Entfaltung. Bei der Absteckung der einzelnen Baukompartimente bediente man sich in Meißen offenbar eines Fußmaßes von 29,03 $\pm 0,5 \mathrm{~cm}$ als Grundlage für die arithmetische Konstruktion der

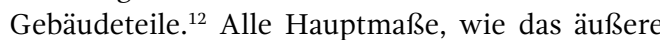
Gesamtmaß des Chores mit 52 x 36 Fuß, das innere Gesamtmaß des Querhauses mit 30 x 96 Fuß, die innere Breite des Chores mit 30,5 Fuß und die Wanddicke von 3,5 Fuß im Chor, hatten dabei möglichst ganzzahlige Vielfache dieses Fußmaßes zu sein, um sie mit einem großen Zirkel abtragen $\mathrm{zu}$ können. Durch die Ungenauigkeiten in der Baudurchführung oder vielleicht durch topografisch bedingte Abweichungen und Ungenauigkeiten, z. B. in der Parallelstellung der Wandkonstruktionen, ist eine konsequente Anwendung dieses Maßsystems jedoch heute nur sehr schwer nachzuweisen. Die Vorgabe eines über alle Bauphasen hinweg beizubehaltenden „Moduls“ war aber unerlässlich für die Durchführung der geometrischen Grundkonstruktionen in Grund- und Aufriss.

Um die Nutzung des romanischen Doms während der Errichtung der neuen Kathedrale nicht zu beeinträchtigen, ordnete man den ersten Bauabschnitt des neuen Chores östlich von Querhaus und Apsis des romanischen Baus an. Durch die mutige Entscheidung, bei der neu zu errichtenden Kathedrale die Größe des romanischen Doms vor allem in seiner Längserstreckung nahezu zu verdoppeln, musste wegen der räumlichen Enge auf

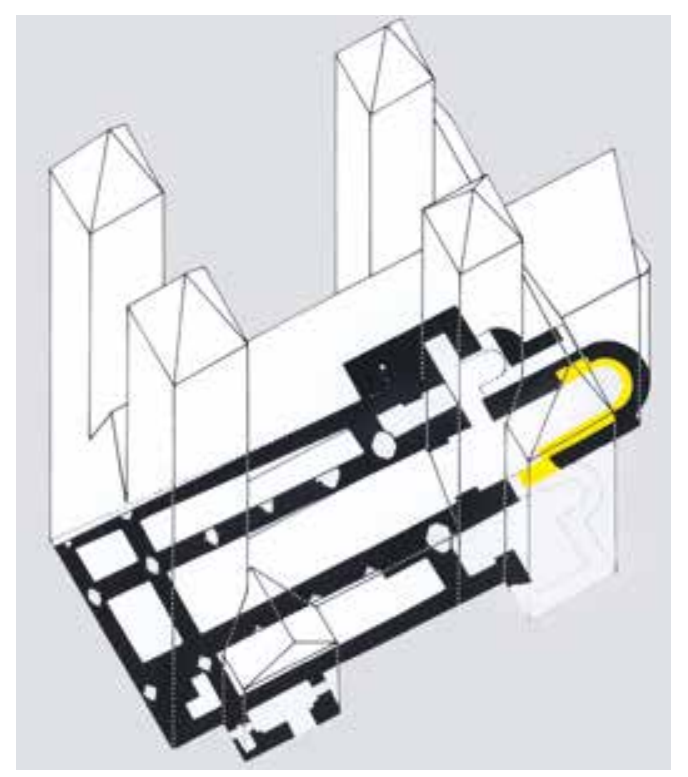

Rekonstruktionsvorschlag für den romanischen Meißner Dom, entwickelt über den 1909 und 1993/94 ergrabenen Baubefunden (schwarz: romanischer Dom, gelb: vorromanischer Saalbau)

๑) Architekturbüro Donath dem im Grundriss etwa dreieckigen Plateau des Dombergs das neue Sanktuarium weit nach Osten über die bestehende Hangkante hinaus ,geschoben" werden. Damit geriet man aber in ein unwegsames und felsiges, nahezu 50 Meter zur Elbe hin steil abfallendes Gelände.

Dorthin musste nun die in mente conceptum manifestierte Bauidee im Maßstab 1:1 in das Baugelände übertragen werden. Man kann nur vermuten, dass dabei zunächst einzelne Hauptpunkte des gotischen Grundrisses vom älteren Gebäude her übertragen wurden. Mit dem Bau der etwa 3,95 Meter tiefen, gemauerten Fundamente der Wandzungen des Stifterjochs und der beiden Treppentürme wurde an der Ostseite des alten Querhauses begonnen und die Fundamentkonstruktionen von West nach Ost in Richtung Steilhang vorangetrieben. Der Aushub erfolgte bis auf Höhe des „Felsknacks“, der noch manuell abzuräumen- 
13 Siegfried Wand: Baugrund und Geologie des Meißner Burgbergs. In: Günter Donath (Hrsg.): Die Restaurierung des Doms zu Meißen 1990-2002. Stuttgart 2003, S. 233-236.

Übersicht über die im Mittelalter üblichen Steinmetzwerkzeuge aus: Robert Habermayer: Mauerwerkstechnik und Steinbearbeitung, 1983 den lockeren Verwitterungsschicht des mit Rhyolithgängen durchzogenen Syenit-Felsstocks. ${ }^{13}$ Die tiefen Gräben mussten verzimmert werden und wurden anschließend mit roh behauenen Sandsteinquadern und einer Kernfülle aus Bruchsteinen ausgemauert. Die Fundamentbreiten waren so geplant, dass sie gegenüber dem Aufgehenden außen nur wenige Zentimeter, auf der Innenseite nur ganz wenig überstanden. In der Achse der Jochbögen wurden sogenannte „Spannfundamente "14 ausgeführt, die beide Chor-Längswände gegeneinander aussteifen sollten. Spätestens mit Erreichen des Sanktuariums musste wegen des steil abfallenden Geländes mit dem Bau der Substruktionen (Unterbauten) begonnen werden. Durch Überwölben der Felsklüfte konnte erst einmal eine Arbeitsebene für das aufgehende Fundamentmau- erwerk geschaffen werden. Zum Aufführen der nachfolgenden Baukonstruktionen mussten von West nach Ost „fortschreitende" Gerüste errichtet werden, von denen aus die Absteckung des komplizierten Chorgrundrisses vorbereitet werden konnte. Immerhin galt es, den über einer horizontalen Ebene entwickelten Grundriß, der fünf Seiten eines Achteckes darstellte, in die Schräge des unwegsamen Steilhanges zu übertragen! Diese polygonale Struktur des Chorhauptes konnte man mit Mitteln der Kreis- und Dreiecksgeometrie konzipieren. Hinweise auf mittelalterliche Vermessungspraxis ergeben sich aus zeitgenössischen schriftlichen Quellen und bildlichen Darstellungen ${ }^{15}$. Demzufolge wurde der Umriss des Meißner Chores mit Gipsmehl oder mit Schnüren auf dem Boden markiert. Hilfsmittel waren Messlatte, Rute, Messschnur, Seile, Pflöcke, rechtwinkliges Dreieck, Zirkel und Lot. Für längere Strecken wurden Messschnüre verwendet, die in 12 Abschnitte unterteilt waren. Rechte Winkel wurden nach den pythagoräischen Zahlen 3, 4 und 5 konstruiert, der rechte Winkel konnte mittels Zirkel halbiert werden. Die Summe beider $\left(135^{\circ}\right)$ bildete den abzusteckenden Innenwinkel des Chorpolygons. Vielleicht wurde aber auch zuerst der abwinkelnde Polygonzug des Chorhauptes markiert, dann später die so ermittelten Linien - wahrscheinlich die Fundament-Außenkanten - genauer mit Hilfe von Seilen und Stangen abgesteckt. An den Eckpunkten des Polygonzuges mussten anschließend unter dem Winkel von $110^{\circ}$ die Wandzungen der künftigen Strebepfeiler angelegt werden. Dabei wurde ein Gründungsniveau am Hang erreicht, das sich etwa 9,75 bis 10,0 Meter unter dem geplanten Chorfußboden als der horizontalen Planebene befand. Durch den gewaltigen Höhenunterschied ragten nun die Substruktionen hoch am Hang hinauf. Deshalb mussten bereits von dieser Tiefe an die talseitig später sichtbaren Konstruktionsglieder aller Unterbauten steinsichtig aus sorgfältig behauenen Sandsteinquadern ausgeführt werden, während man den hangseitigen „Rücken“ dieser Wände mit Rohbossen und Bruchsteinen ausmauern konnte. Mit dem Erreichen des geplanten unteren Chorumgangs konnte nun das aufgehende Mauerwerk des Chorpolygons aufgerissen werden. Dazu wurde sicher ein Netz von sich auf die Hauptachsen des Bauwerks beziehenden Konstruktionshilfslinien in die Steinplatten des Umganges geritzt, so wie diese auch auf dem Laufgang des Naumburger Westchores nachgewiesen werden konnten. ${ }^{16}$

Die Dokumente der Domgrabung von 1910 zeigen, dass die Fundamente innen knapp unter den Wandkonstruktionen sitzen, deren unterste Schicht wiederum genau auf dem Fundamenthaupt ausgerichtet ist. Nach außen hingegen ist ein breiterer Fundamentüberstand festzustellen. Das hat nicht zuletzt mit statische Gründe: Da die Resultierende aus dem Gewölbeschub innerhalb der Strebepfeiler schräg nach außen verläuft, besteht die Gefahr, dass sie dicht unter der Erdober- 
fläche aus dem Fundament heraustritt, wenn dieses nicht ebenfalls nach außen verbreitert wird. Dieser Zusammenhang wurde von den alten Baumeistern zwar nicht rechnerisch erfasst, wohl aber empirisch gewusst und entsprechend berücksichtigt.

Über den profilierten Sockel hinweg wurden nun die hoch aufstrebenden Wandkonstruktionen zunächst bis in Höhe der Fensterbänke aufgeführt. Den Anschluss an die östlichen Querhauswände des romanischen Doms bildete eine stehende Verzahnung. Bereits in diesem ersten Bauabschnitt lassen sich baumeisterliche Überlegungen für ein effizientes Bauen nachweisen: Während man unterhalb der Ebene des Chorfußbodens außen bereits mit sorgfältig bearbeiteten Sandsteinquadern arbeiten musste, wurde die innere Wandseite noch ganz sparsam mit Rohbossen errichtet. Erst oberhalb des Fußbodenniveaus musste das zweischalige Mauerwerk innen und außen in höchster Qualität ausgeführt werden. Dabei wurden die innere und die äußere Mauerschale getrennt voneinander aus etwa 28 bis 42 Zentimeter tiefen Sandsteinquadern errichtet, die an fünf Seiten regelmäßig bearbeitet sind. Den Zwischenraum füllte man mit Hausteinabfällen, Bruchsteinen und Mörtel aus. Die Lagerfugen der beiden Mauerschalen befinden sich außer in der Fuge über dem Sockel und unterhalb der Fensterbänke in der Regel nicht auf gleicher Höhe. Die Höhen der einzelnen Steinlagen und damit die Abstände der Lagerfugen schwanken; sie ergaben sich einerseits aus dem Bestreben, die angelieferten Rohblöcke bzw. im Steinbruch grob zugerichteten Steine möglichst wirtschaftlich auszunutzen, andererseits aus im Bauplan festgelegten Niveaus, die - einmal eingemessen - dann leicht mittels Setzwaagen über große Abschnitte hinweg einzuhalten waren, wie etwa den Höhen des Sockels und der Fenstersohlbänke. Die Lagerfugen ziehen sich konsequent bis in die Fenstergewände und Dienste mit ihren komplizierten Profilfolgen und in die Strebepfeiler hinein. Mit hohem Sachverstand wurde differenziert zwischen dem weicheren und damit leichter zu bearbeitenden, aus den Niederschönaer und Grillenburger Brüchen stammenden Steinmaterial für den Innenraum und dem festen, quarzitisch gebundenen Sandstein mit hoher Festigkeit und Verwitterungsbeständigkeit für die äußere Schale und die Strebepfeiler. ${ }^{17}$

Für die Herstellung ästhetischer Steinansichtsflächen verwendete man große Mühe. Die Steine wurden zunächst mit dem Zweispitz oder der Pille grob bearbeitet, dann mit der Glattfläche, einem Hiebwerkzeug in Form eines Doppelbeils, das die Herstellung einer glatten Oberfläche des Quaderspiegels ermöglichte.

Auf der Steinoberfläche zeichnet sich diese Bearbeitung durch parallele, leicht unregelmäßige Hieblagen aus. Die Hiebrichtungen können sich von Stein zu Stein unterscheiden, auch verlaufen die Hieblagen nicht unbedingt streng parallel. Man erzielte dadurch eine belebte Oberfläche. An den

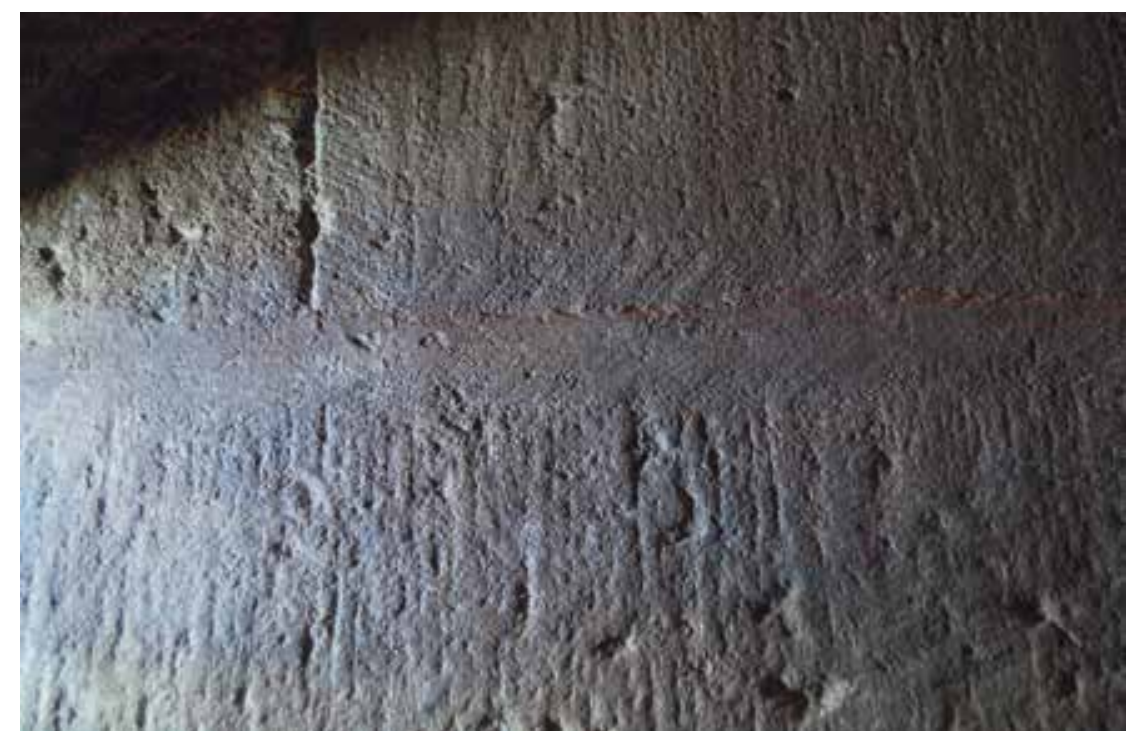

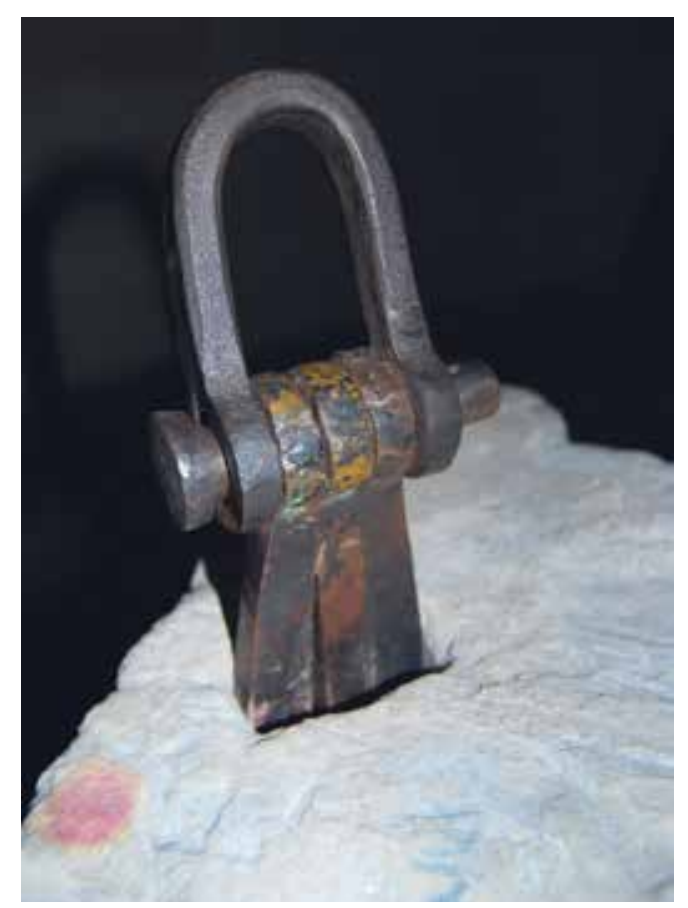

Rändern ist mitunter ein sauberer, etwa $2 \mathrm{~cm}$ breiter Randschlag sichtbar, der an den ersten Schritt des Arbeitsprozess erinnert. Der Steinmetz definierte zuerst die Ecken des Steinquaders und bearbeitet dann die Ränder der Sichtfläche in Längsrichtung, bevor er die Frontseite mit der Glattfläche glättete. Bei der Feinbearbeitung der Sichtfläche blieb der Randschlag teils stehen, teils wurde er abgearbeitet. Versetzt wurden die an fünf Seiten bearbeiteten Steinquader entweder mit dem Wolf, einer auf der Steinoberseite in Schwerpunktlage angebrachten Aufhängevorrichtung, welche in einer schwalbenschwanzförmigen ausgearbeiteten Vertiefung mit einem mehrteiligen, der Schwalbenschwanzform folgenden Eisenkeilen gehalten wurde, oder mit der Steinzange, die mit ihren gewaltigen geschmiedeten Schenkeln den Stein von oben umgreift und deren Enden in dafür ausgespitzten Zangenlöchern Halt finden.
Quaderspiegel mit Randschlag im unteren Chorumgang des Meißner Doms

Foto: Günter Donath

Bei Grabung geborgener Werkstein aus dem im 14. Jahrhundert abgebrochenem Lettnerziborium des Doms mit Wolf im Wolfsloch Foto: Günter Donath

14 Der in der Archäologie übliche Begriff „Spannfundament“ ist aus baustatischer Sicht Unsinn, da die in der Regel aus Bruchsteinen errichteten Fundamente keine Zugkräfte übertragen und demzufolge auch keine Zugspannungen aufnehmen können. Sie werden druckbeansprucht und steifen die Längswände gegeneinander aus.

15 Günter Binding: Mittelalterlicher Baubetrieb. Darmstadt 1993, S. 339.

16 Vgl. Donath/Donath 2011 (wie Anm. 6)

17 Donath/Donath 1998 (wie Anm. 8), S. 194-211. 


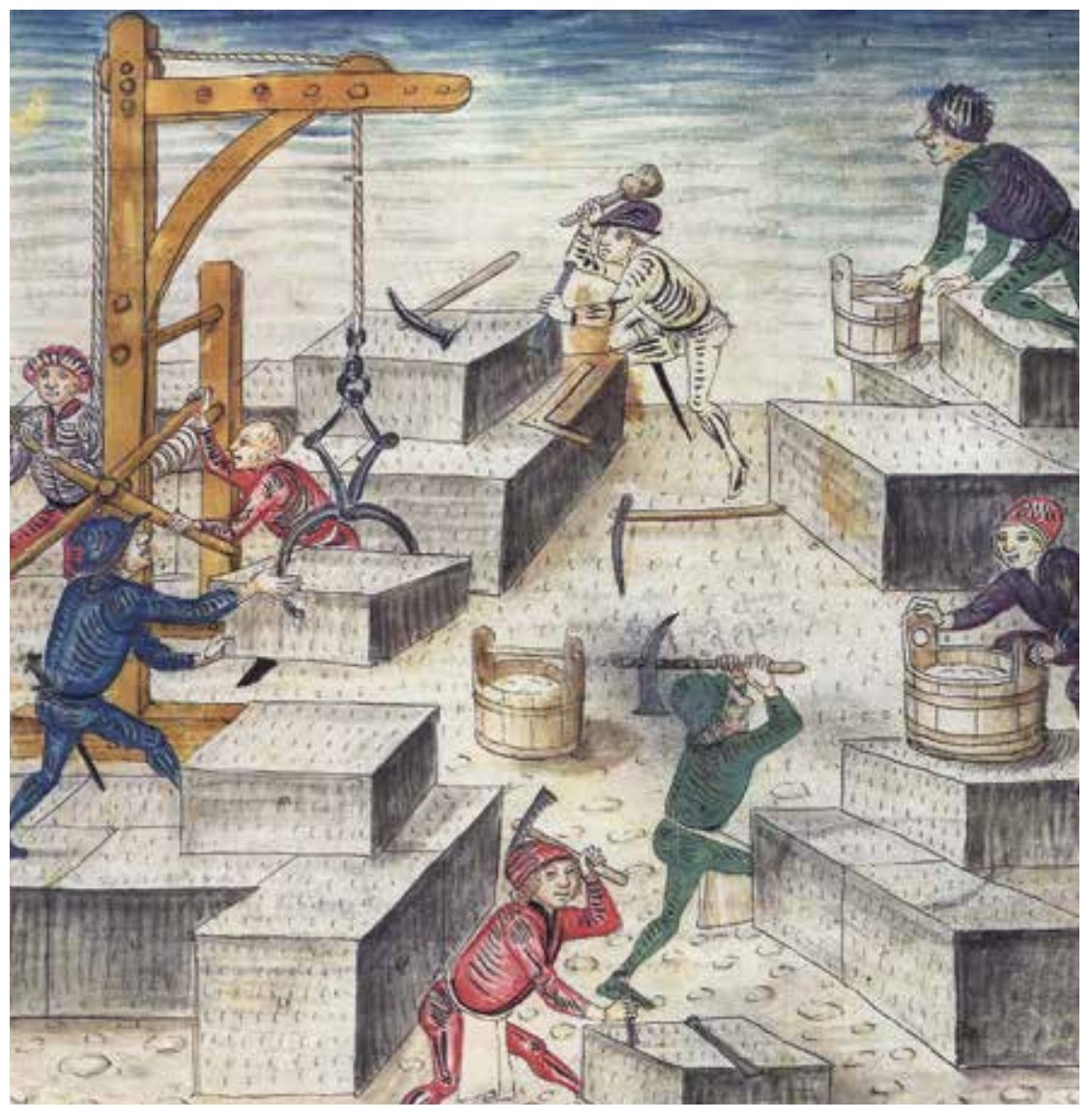

Steinmetzen auf dem Werkplatz, Galgenkran mit Haspel und Steinzange aus: Diepold Schilling, Amtliche Berner Chronik 1478, Bern, Stadtbibliothek Mss.hist.Helv.l,1,225.

Isometrische Darstellung der ersten Bauphase (la) des gotischen Neubaus Zeichnung: Architekturbüro Donath

Mit dem Erreichen der Höhen für die geplanten Fensterbänke ( 9 Schichten) bzw. bei ca. $+5,70$ Meter über der Oberkante des Fußbodens (13 Schichten) endete der erste Teilbauabschnitt. In dieser Höhe kann man - beginnend an den stehen-

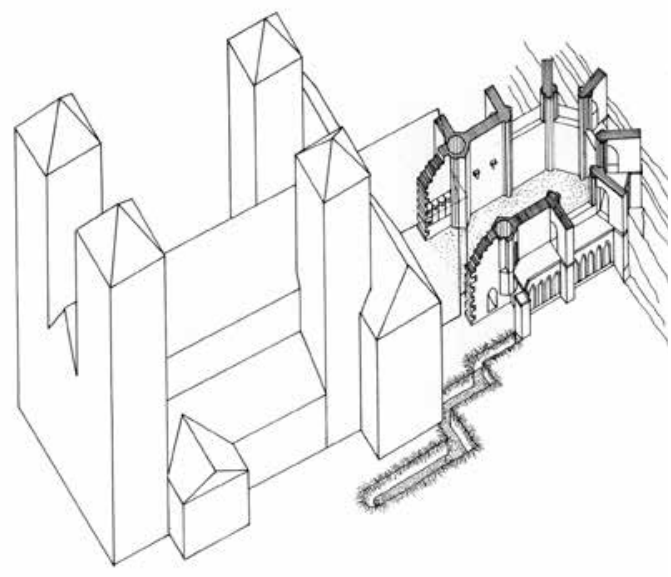

den Verzahnungen am Westende dieses Bauabschnittes - eine durchgehende, vertikal verlaufende Baufuge beobachten. Talseitig hatte man damit gleichzeitig die Arkatur an der Außenseite des Chorumgangs (unterer Laufgang) mit aufgeführt. Dieser Umgang wurde mit Steinplatten abgedeckt. Damit war nicht nur die Ebene für einen oberen Laufgang entstanden, sondern gleichzeitig eine neue Arbeitsebene in 14 Meter Höhe über dem Wandfuß geschaffen worden, von der aus nun die Gerüste für das Aufgehende des nächsten Teilbauabschnitts gestellt werden konnten. Auf Grund der gewaltigen vermessungstechnischen und konstruktiven Leistung muss man davon ausgehen, dass hier als Baumeister kein Anfänger am Werke war. Woher hatte er aber seine enormen Fähigkeiten? Kannte er solche komplizierten Gründungsbauwerke, wie sie der Architekt Robert de Luzarche in Amiens errichtete oder hatte er Erfahrungen in Köln mit 12 Metern Gründungstiefe, in Magdeburg oder Marburg sammeln können? Wir wissen es nicht.

Betrachtet man diesen ersten Teilbauabschnitt (Ia) mit seiner gestaffelten Tiefe der Fundamentsohle bei (Ia 3) -9,75 Meter über (Ia 2) -8,13 Meter bis (Ia 1) -3,95 Meter und einer Baustellenlänge von etwa 22 Meter bis hin zu einem angenommenen horizontalen Abschluss bei +- 0,0 Meter, so wird rasch klar, welche Massen allein für die Fundamentmauern zu bewegen waren:

Ia 1: für das Strebesystem Nord und Süd, die Füllmauern dazwischen, die Spannfundamente, die beiden Treppentürme mit den Mauerzungen zum romanischen Dom sowie für die Erweiterung des unteren Umgangs an der Südseite zus. $333 \mathrm{~m}^{3}$

Ia 2: für die beiden Polygonpfeiler Nord und Süd mit Füllmauern zus. $144,6 \mathrm{~m}^{3}$

Ia 3: für die beiden östlichen Polygonpfeiler mit ihren Füllmauern

$$
\frac{\text { zus. } 275,5 \mathrm{~m}^{3}}{753,1 \mathrm{~m}^{3}}
$$

Zum betrachteten Bauabschnitt gehört noch das Quaderwerk bis zu einer angenommen Höhe von $+5,70$. Dafür sind anzusetzen in den einzelnen Baulosen:

Ia 1 + Ia 2: die gesamte Chor-Nordseite zus. $179,1 \mathrm{~m}^{3}$ die gesamte Chor-Südseite zus. $168,8 \mathrm{~m}^{3}$

Ia 1: das Chorpolygon mit dem Laufgangsystem zus. $186,7 \mathrm{~m}^{3}$ $534,6 \mathrm{~m}^{3}$

Kubatur gesamt: $1.287,7 \mathrm{~m}^{3}$

Die Baumassen haben zusammen ein Gewicht von rund 3.100 Tonnen. 78 Prozent davon - das sind 1.004 Kubikmeter - waren Rohbossen oder behauene Quader, 15 Prozent = 193 Kubikmeter war- 


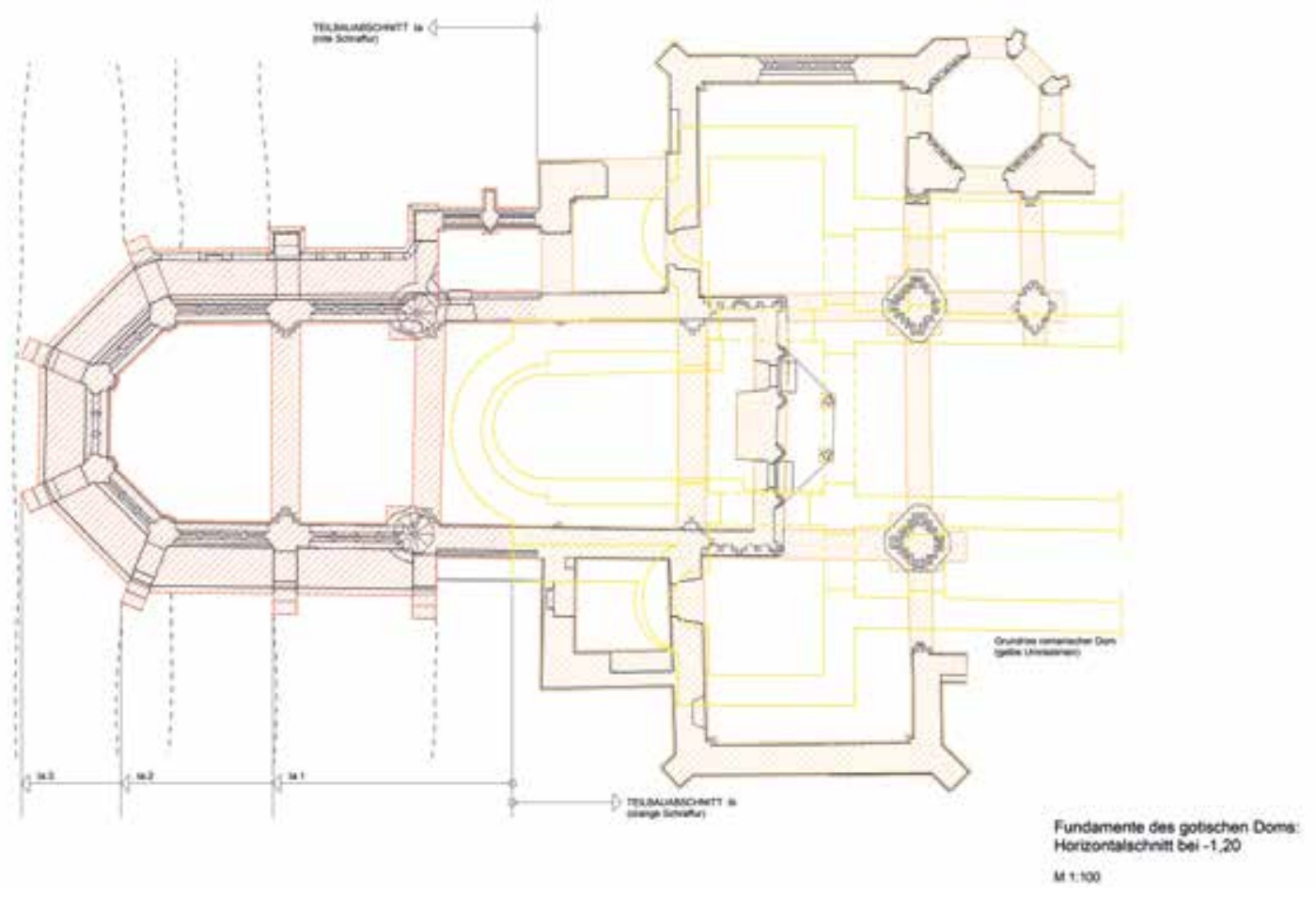

en Bruchsteine für das Ausmauerungsmaterial. Um diese Menge zu vermauern mussten $64.400 \mathrm{Li}$ ter Mörtel Kalk eingesumpft, dann gemischt und vermauert werden. Der Sand kam aus den im Raum Kagen und Taubenheim gelegen Gruben. Es musste langsam gemauert werden, um die Steinschichten der Fundamente besser abbinden zu lassen. Beim Bau der Fundamente für den Kölner Dom $^{18}$ ließ sich unter vergleichbar komplizierten Bedingungen ein Bautempo von ca. 4,2 Kubikmeter pro Tag nachweisen. Die dafür benötigte Menge an Quadersandstein entspricht auch der, die mit einer Schiffsladung täglich herangeschafft werden konnte. Mit diesem Tempo konnte eine Versetzerkolonne täglich 13 bis 15 Quader vermauern. Rechnet man wegen der zahlreichen kirchlichen Feiertage das Jahr mit 180 Arbeitstagen, so kommen allein für die logistischen Vorbereitungen wie das Einrichten der Baustelle, den Gerüstbau für die Absteckung und das Mauern der aufragenden Substruktionen, den Aushub der Fundamentgräben, das Verzimmern und das Herstellen der Fundamentmauern, das Verfüllen eine Bauzeit von 11/2 Jahren zusammen. Hinzu kommt noch ein weiteres Jahr für das Aufführen des Chores bis zur Höhe +5,70 Meter, also insgesamt 2 1/2 Jahre Bauzeit allein für den Teilbauabschnitt Ia.

\section{Planung und Bauausführung}

des Teilbauabschnitts Ib

Um den Chorhals mit den beiden Osttürmen und das Querhaus weiter ausführen zu können, mussten zuerst die Hauptapsis und das Querhaus einschließlich der beiden Osttürme des romantischen Doms bis auf die Höhe des zweiten Joches von Ost des basilikalen Langhauses abgetragen werden. Zuvor hatte man einen provisorischen Giebel - wahrscheinlich als Fachwerkwand eingezogen, damit das Domkapitel den für Chorgebete und Messen notwendigen Raum im Westen der alten Domkirche beziehen konnte. Anschließend galt es aber, den gotischen Neubau rasch voranzutreiben, denn es war wohl das oberste Ziel bei allen Baumaßnahmen, möglichst schnell nutzungsfähige Baukompartimente entstehen zu lassen. Der Dombaumeister versuchte durch zwei Maßnahmen, das Bautempo zu erhöhen: er verstärkte schlagartig die Zahl der Steinmetzen in der Hütte, so dass künftig mindestens zwei unabhängig voneinander agierende Versetzerkolonnen am Bau arbeiten konnten, und er änderte die Technologie des Steinversatzes. Der anfangs verwendete Wolf mit dem sehr zeitaufwändigen Steinversatz musste zugunsten eines größeren Bautempos dem viel schnelleren und wirtschaftlicheren Versatz durch die Steinzange weichen. ${ }^{19}$ Die damit verbundenen sichtbaren Zangenlöcher in den Quaderspiegeln scheinen das ästhetische Empfinden nicht gestört zu haben.

Zuerst wurden sicher die Ausführung der beiden Osttürme mit ihren Anschlüssen an die Wandzungen des ersten Bauabschnittes und die beiden langgestreckten Chorhalswände mit dem Dorsale, der steinernen Rückwand des Chorgestühls, und dem Lettner in Angriff genommen. An den Osttürmen mussten bereits die Anschlüsse der Querhauswände vorbereitet werden. Die Sohltiefe der Ausschachtungen für die Fundamente wurde zumindest bei den Türmen mit -3,95 Metern beibehalten. Zwischen die Chorhalswände wurde ein weiteres Spannfundament eingezogen. Der sich westlich anschließende Kernbau des Lettners erhielt einen eigenen Fundamentblock, während
Rekonstruierter Fundamentplan des Bauabschnittes bis 1266 Zeichnung: Architekturbüro Donath

18 Alle Vergleichszahlen aus Wolff 1968 (wie Anm. 7).

19 Einen Wechsel des Baumeisters daraus abzuleiten - wie Katja Schröck: Darstellung und Spuren des Steinmetzgeschirrs. In: Bruno Klein/ Stefan Bürger (Hrsg.): Werkmeister der Spätgotik. Personen, Amt und Image. Darmstadt 2010, S. 26-44, vermutet - ist jedoch nicht zu begründen und lässt sich auch nicht nachweisen. 
Vertikalschnitt durch den Hohen Chor des Meißner Doms mit Blickrichtung Süd Zeichnung: Architekturbüro Donath

20 Den bisherigen Forschungsstand $\mathrm{zu}$ mittelalterlichen Ritzzeichungen fasste Wolfgang Schöller zusammen, vgl. Schöller, Wolfgang: Ritzzeichnungen. Ein Beitrag zur Geschichte der Architekturzeichnung im Mittelalter. In: architectura. Zeitschrift für Geschichte der Baukunst 18 (1989), S. 36-61. Dem Katalog sind die neu entdeckten Zeichnungen in Meißen, Naumburg und Schulpforta anzufügen.

Werkriss an der Südwand des Hohen Chores, farbige Nachzeichnung des Befundes (rot = Hauptriss für die Geometrie der Polygonpfeilers, gelb = Binnenzeichnung für die Durchbildung der einzelnen Steinlagen sowie Hilfslinien, schwarz = nachträglich ergänzte Konstruktionshilfslinien für die Goldene-SchnittTeilung einer Strecke) Foto und zeichnerische Bearbeitung: Architekturbüro Donath

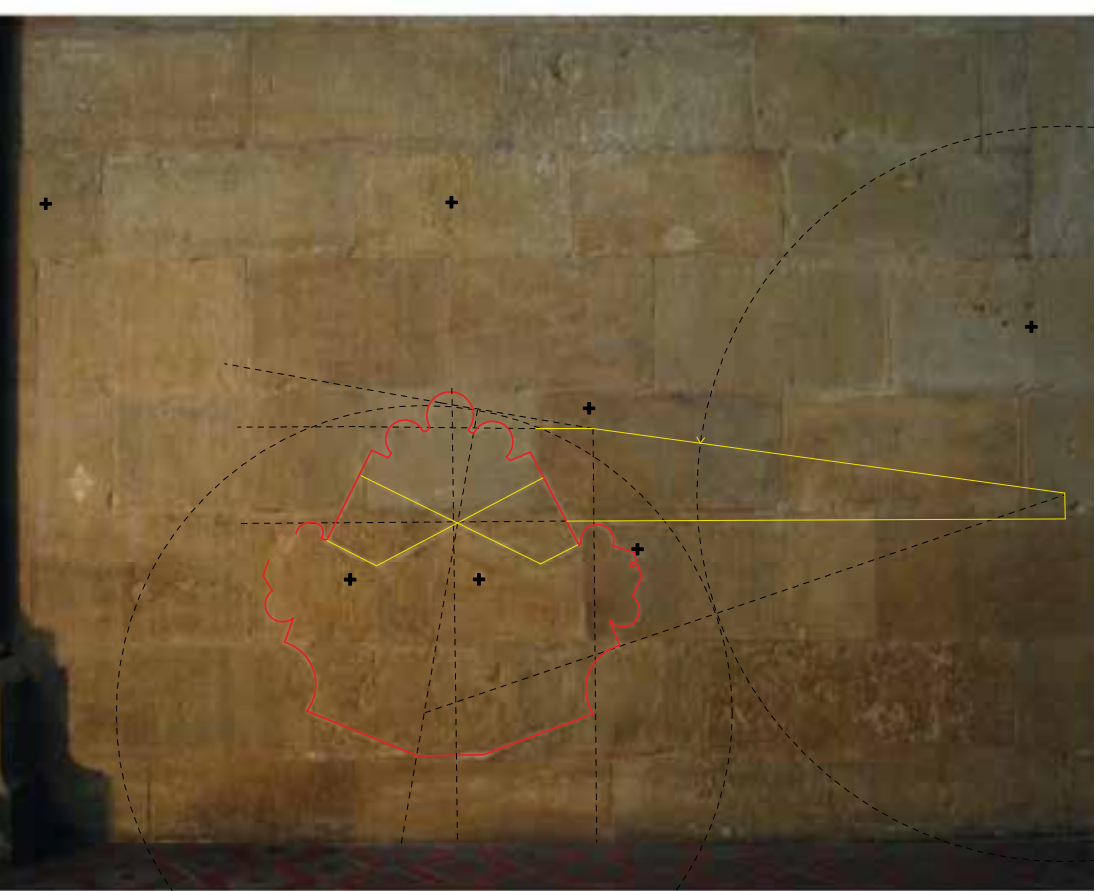

die beiden ein Ziborium tragenden Pfeiler auf kleine quadratische Punktfundamente abgesetzt wurden. Zwischen den Chorhalswänden ließ man die Fundamente des romanischen Doms, der bis etwa $40 \mathrm{~cm}$ unter das Niveau des Chorfußbodens abgetragen wurde, einfach liegen. Die Fundamentmauern, mit deren Bau erst nach Abbruch des romanischen Doms begonnen werden konnte, umfassen:

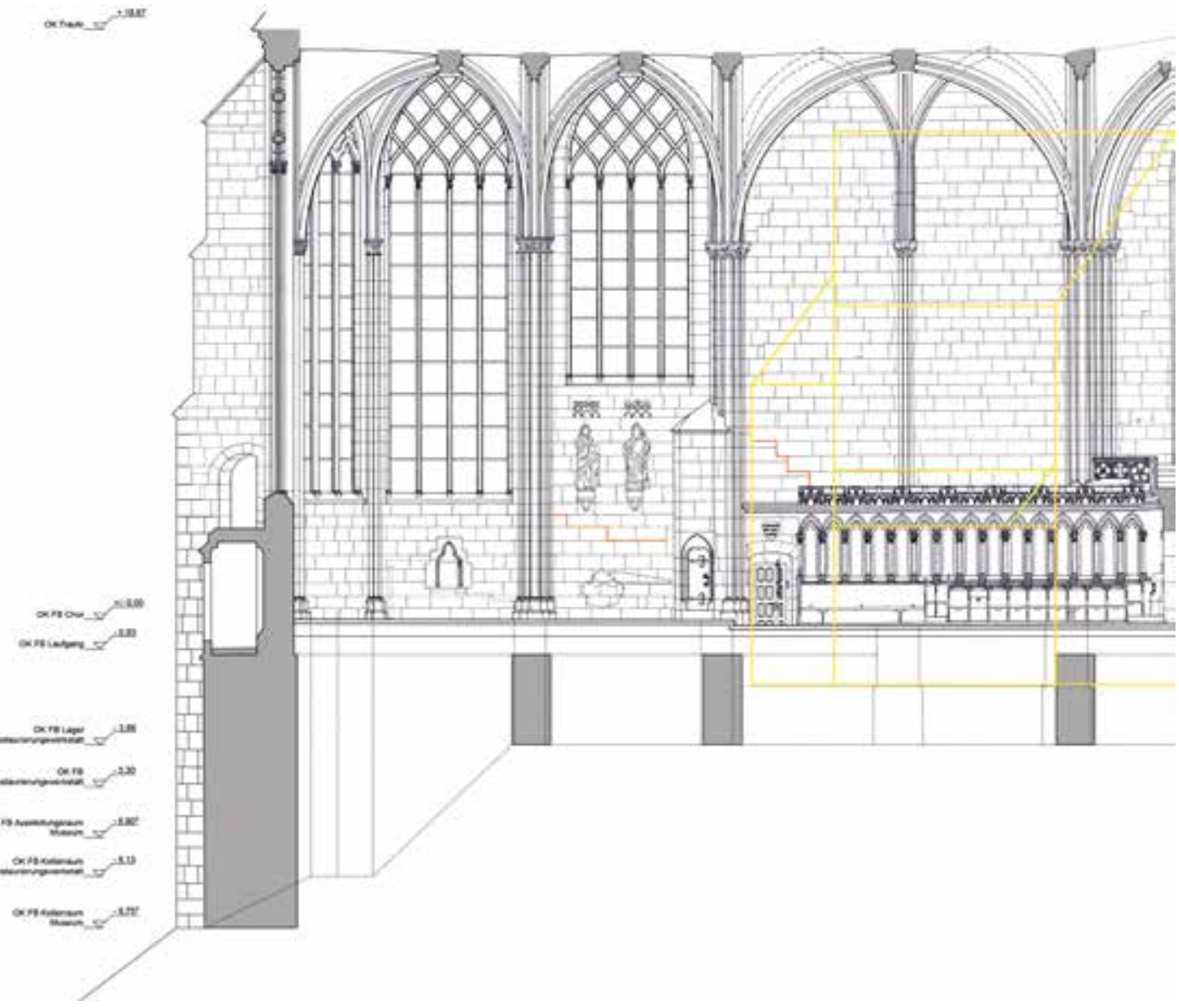

Chorflankentürme zus. $\quad 200 \mathrm{~m}^{3}$ Spannfundamente der Vierung mit Lettner zus. $54,8 \mathrm{~m}^{3}$ Nordquerhaus zus. $71,4 \mathrm{~m}^{3}$ Südquerhaus mit Achteckbau und basilikalem 1. Joch zus. $117,1 \mathrm{~m}^{3}$ ein Volumen von $443,3 \mathrm{~m}^{3}$

Insgesamt ist dafür eine weitere Bauzeit von einem Jahr anzusetzen. Die dann aufzuführenden Quaderwände waren künstlerisch äußerst anspruchsvoll: Wie in Naumburg zierten ein steinernes Dorsale und der Lettner mit einer überaus reichen Architekturgliederung durch Blendarkaden, Kapitellen mit floralen Darstellungen, Baldachinreihen und Laubwerksfriesen den unteren Bereich des Aufgehenden. Hier finden sich nur drei Steinmetzzeichen, von denen eines am Lettner wiederkehrt. Erst mit Erreichen der Höhe $+5,70$ Meter, der Mauerwerksoberkante des Abschnitts Ia, konnten dann die Schichten ganz im Sinne des Konzeptes der Lagerfugenbauweise vom östlichen Triumphbogen über Chorhals und Chorpolygon um den gesamten Chor auf gleicher Höhe durchlaufen. Spätestens zu diesem Zeitpunkt musste die Ausführung so wichtiger Baudetails, wie sie die statisch unverzichtbaren, weil den Chorbau aussteifenden Polygonpfeiler mit den unmittelbar an ihren Flanken ansetzenden Fensterleibungen und 
den raumseitigen Diensten darstellen, klar sein. Auf der Südwand des mittleren Chorjoches wurde im Maßstab 1:1 der Horizontalschnitt durch einen Polygonpfeiler mit allen dazu gehörenden Konstruktionshilfslinien in die Quaderspiegel der Mauer eingeritzt. In ganz Europa sind bisher nur etwa 50 solcher „Werkrisse“ genannter Konstruktionszeichnungen der mittelalterlichen Baumeister bekannt geworden. Die Ritzzeichnungen ermöglichen einen Einblick in das Baugeschehen im 13. Jahrhundert. ${ }^{20}$ Die meist im Maßstab 1:1 dargestellten Gebilde waren keine Freihandzeichnungen, sondern geometrisch konstruiert und stellen ein gotisches Zeichnungskonvolut dar - nicht auf Pergament oder Papier, sondern eingeritzt in die Sockelwände des entstehenden Bauwerks.

Die mittelalterliche Bauhütte nutzte also den noch unfertigen Chor als „Planungsbüro“ und Werkstatt. Dort - inmitten des turbulenten Baubetriebs, wo Material angeliefert, die Quader behauen und versetzt, die Hausteinabfälle zusammen mit dem Kalkmörtel in das Wandinnere gefüllt wurden arbeitete man auch den Entwurf im Detail aus. Direkt auf der Wand wurde geplant, entworfen und offenbar auch wieder verändert. Die Steinmetze fertigten nach diesen Zeichnungen hölzerne Schablonen an, die sie für das Aufreißen der Werksteine auf den Rohblöcken verwendeten. Die Werkrisse sind wegen der Überlagerung der Lineatur mitunter nur dünn eingeritzt und nur wenig anschaulich. Ein wichtiges Merkmal sind die oft noch zu erkennenden Einstichlöcher des Zirkels, mit dem Kreisbögen und Strecken abgetragen wurden. Der gotische Werkmeister nutzte Metallgriffel, Richtscheit und Zirkel, um diese Werkrisse geometrisch zu konstruieren. Mitunter wurde die Steinoberfläche dabei eingefärbt, um die Ritzungen besser hervorzuheben. ${ }^{21}$ In Meißen findet man darüber hinaus noch die Abtragung des am Dom verwendeten Fußmaßes von $29 \mathrm{~cm}$ Länge mit einem markanten Zirkeleinstichloch sowie den beim Bau der französischen Kathedralen üblichen „Pariser Königsfuß“ von $32 \mathrm{~cm}$ Länge. ${ }^{22}$ Bemerkenswert ist, dass sich auch in Naumburg an gleicher Stelle ein Werkriss befindet, dort mit einem Detail des Westlettners $^{23}$ - und ist nur eines von vielen Beispielen, mit denen sich gleiche Baugewohnheiten auf diesen beiden Baustellen belegen lassen.

Von diesem Zeitpunkt an konnten die Versetzerkolonnen unabhängig voneinander arbeiten: die eine am Aufgehenden des Chores und der Osttürme, die andere mit dem Bau der Fundamente und des Aufgehenden von Querhaus mit dem Achteckbau, einem zweigeschossigen turmartigen Eingangspavillon im Winkel zwischen dem südlichen Querhausarm und dem Südseitenschiff des künftigen Langhauses. Mit diesem in unmittelbarem baulichen Zusammenhang stehend wurde innen auch das erste basilikale Seitenschiffsjoch errichtet. Beim Anlegen des nördlichen Querhausarms musste Rücksicht auf die nördlich des Domes befindlichen Gebäude der Markgrafenburg genommen werden. Deshalb weicht dessen Grund-

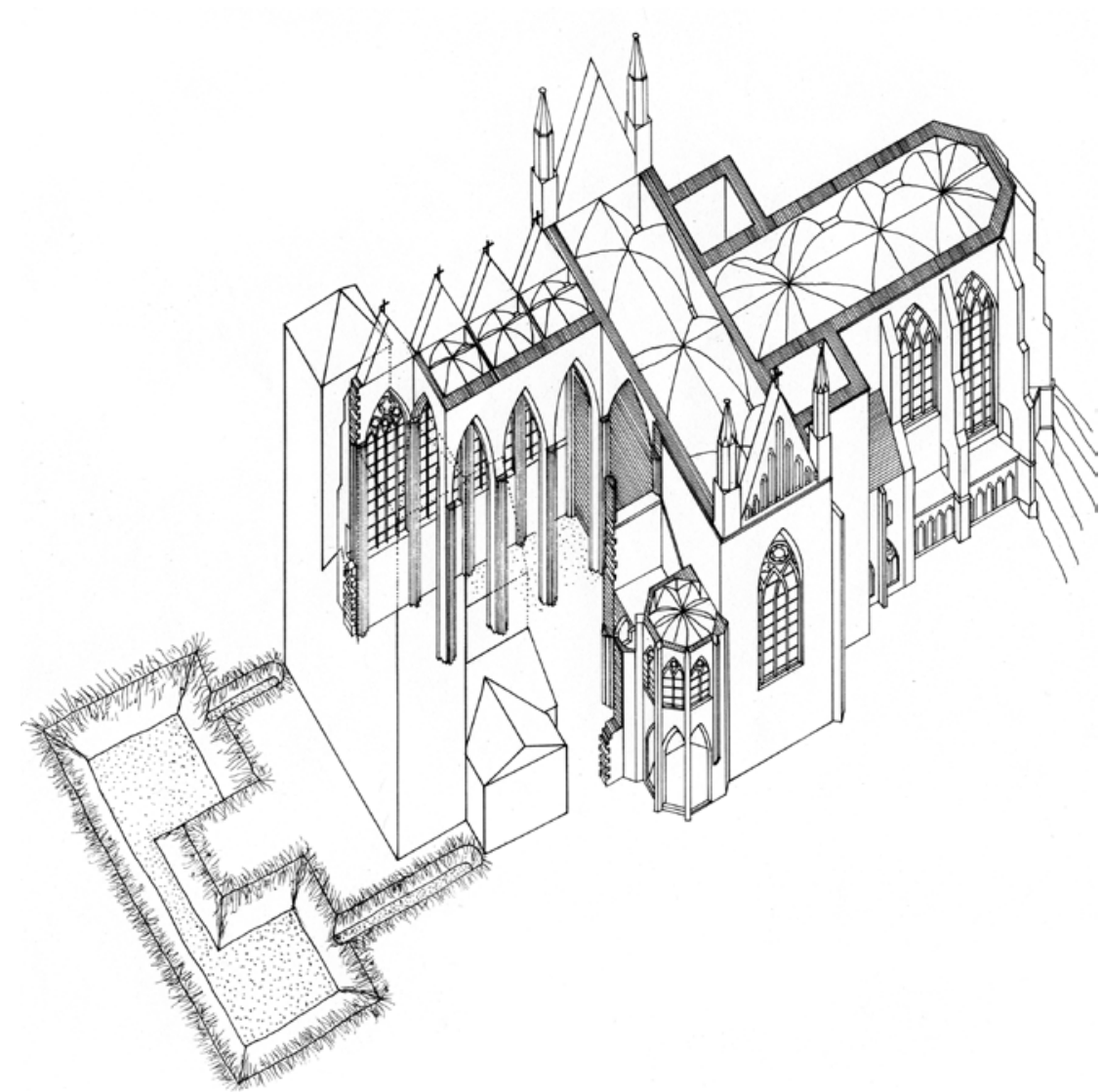

riss vom quadratischen Schema ab und ist nach Norden hin eingekürzt. Auch beim Südquerhaus sind Unregelmäßigkeiten zu beobachten: Die südöstliche Querhauswand schließt innerhalb des südöstlichen Vierungspfeilers nicht im rechten Winkel an die Chorhalswand an. Dieses „Herausdrehen" ist nur minimal und offenbar einem Messfehler und einer zusätzlichen Verdrehung beim Anlegen der Rippenverläufe im Kämpferblock des Vierungspfeilers geschuldet. Welche Informationen die Baumeister an der Oberfläche eines Kapitellblockes anbrachten, wissen wir durch die Untersuchung des wahrscheinlich 1910 ausgebauten Kapitells, das sich heute im Hof der Dompropstei befindet. Die durch fehlerhaftes Aufreißen bedingte Differenz musste aber beim weiteren Aufmauern wieder ausgeglichen werden und hatte eine Verkröpfung der Rippe im Schildbogen der Ostwand zur Folge. Auch gegenüber der Südwestecke des Querhauses ist der in diese Achse gehörende südwestliche Vierungspfeiler leicht disloziert. Immer ziehen die Ungenauigkeiten im Aufgehenden Probleme in der Wölbung nach sich. Der alte Streit, ob gotische Grundrisse durch figurale Geometrie oder durch modulare Arithmetik erzeugt wurden, ist für die Ostteile des Meißner Doms also nur salomonisch zu schlichten: „Dem geometrischen Wollen folgte die Umsetzung in kommensurablen Größen auf dem Bauplatz ${ }^{24 “}$.

Bis zum Erreichen des Hauptgesimses bei $+18,9$ Meter waren umlaufend 43 Schichten des Quadermauerwerks aufzuführen. In ihnen sind enthalten: die Dienste und Kämpferkapitelle, der Gewölbean-
Isometrische Darstellung des gotischen Neubaus mit den nach einem Planwechsel als Halle gebauten drei Jochen des Nordseitenschiffes, während an der Südseite noch das erste basilikale Joch der Bauphase lb (1250 bis 1266) zu sehen ist.

Zeichnung: Architekturbüro Donath

21 Ulrike Heckner: Die Entwürfe von Magister Enghelbertus für das gotische Fenstermaßwerk in der Chorhalle des Aachener Münsters. In: In situ. Zeitschrift für Architekturgeschichte 1 (2009), Heft 2, S.193-204.

22 Vgl. Günter Donath: Werkrisse. Zeugnisse werkmeisterlicher Planungen auf der Albrechtsburg und im Dom zu Meißen. In: Monumenta Misnensia. Jahrbuch für Dom und Albrechtsburg zu Meißen 9 (2009/2010), S. 121.

23 Vgl. Donath/Donath 2011 (wie Anm. 6).

24 Zitiert nach Norbert Nussbaum: Beobachtungen zur gotischen Konstruktion an der Zisterzienserkirche Altenberg. München 2008, S. 12. 
25 Klaus Pieper: Die Entwicklung der Technik des Gewölbebaus in Deutschland in der Zeit zwischen 1050 und 1350. In: Abhandlungen der Braunschweigischen Wissenschaftlichen Gesellschaft 43 (1992), S. 253.

Hohen Chorfiguren: die Stifter Kaiser Otto I. der Große und seine Gemahlin Adelheid sowie die Bistumspatrone Johannes Evangelist und der heilige Bischof

Donatus; Rekonstruktion der ersten Fassung (um 1260) nach Befund; Farbauslegung der Zeich-

nung durch Restaurator Peter Vohland, Hochstift Meißen, 1996 ๑ Hochstift Meißen
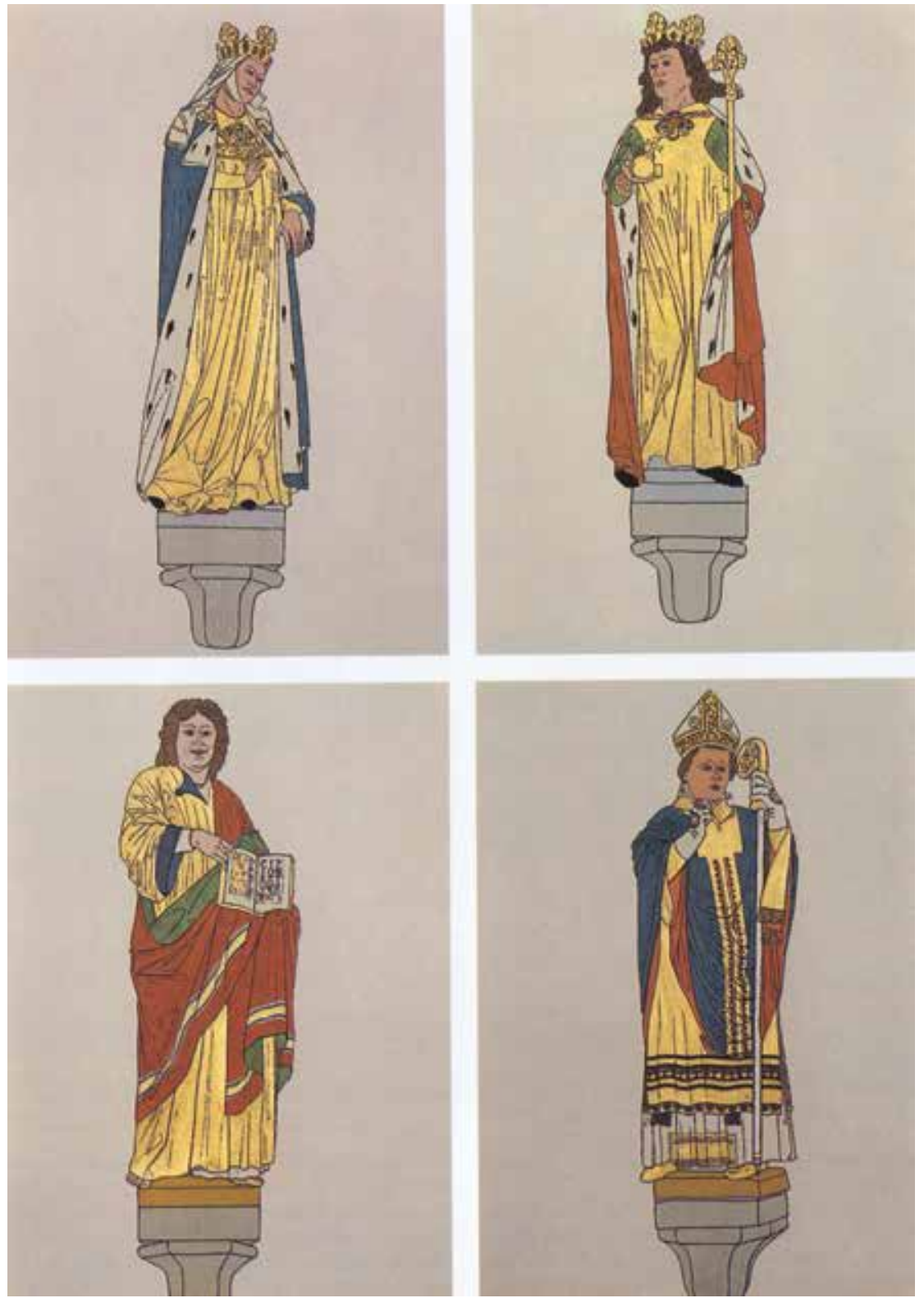

merten Lehrbögen und -gerüsten versetzt. Das Mauern der Einwölbung mit Backsteinen erfolgte dann im „Freivorbau“25 von Rippe zu Rippe bei extremer Reduzierung der sonst üblichen Kappenstärke. An den Unterseiten der Backsteine konnten nach dem Abnehmen vermorschter älterer Putze keinerlei unter den Steinkopf gedrückte Mörtelreste mit Schalbrettabdrücken gefunden werden. Damit gehören die Meißner Gewölbe zu den frühen Beispielen einer Technologie, die vor allem erst mit den stark gebusten Gewölben der Backsteingotik zur vollsten Entfaltung reifte.

Mit den Wänden wurden nicht nur gleich die Schild- oder Scheidbögen aufgemauert, sondern auch oberhalb der Kapitellzone die ersten drei Steine der Kreuzrippen mit versetzt. Sie waren als Kragsteine ausgebildet und boten zugleich den Ansatz für die Lehrbögen der Gewölberippen und Gurtbögen. Oberhalb der Schildbögen wurden nur noch die äußeren (sichtbaren) Wandbereiche in Quaderwerk bis zum vorkragenden und stark profilierten Traufgesims aufgeführt, während die innen liegenden Wandbereiche weniger sorgfältig in Bruchstein ausgeführt werden konnten; diese Zone war ohnehin nur vom Dachboden aus zu sehen. Nach dem Aufsetzen des Dachgerüstes, dem Abbund der Gebinde und Gespärre und dem Eindecken der Dächer war der Bau „unter Dach und Fach“, und es konnte mit dem Einwölben der von der Grundrisskonzeption her angelegten Joche begonnen werden. Auf Lehrbögen wurden die vorgefertigten Kreuzrippen und Gurtbögen versetzt. Die Kreuzrippen in Meißen sind durchweg leicht spitzbogig und damit aus statischer Sicht den runden Bogenformen der Romanik verwendeten Bogenformen überlegen, weil sie der statisch idealen Kurve der Parabel am nächsten kommen. Der Baumeister kannte genau die Vorzüge und Schwächen der Spitzbögen; die Schwächen lagen dort, wo die Bögen der Parabel am unähnlichsten waren, also an der Spitze und im unteren Bogendrittel. Dem Prinzip des Bauens mit Auflasten folgend wurden am höchsten Punkt die nahezu tonnenschweren Schlusssteine versetzt, um ein Ausbrechen der Bögen nach oben zu verhindern. Wenn die Bögen eines Jochs standen, so benötigte man keine Schalgerüste mehr; die Zimmerleute und Steinmetze konnten sich dem nächsten zuwenden und die freihändige Aufmauerung der Kappen zwischen den Rippen den Maurern überlassen. Die Technologie des freihändigen Aufmauerns war zuletzt in der Bauforschung nur gebusten Gewölben zugestanden worden, während sich bei allen ungebusten Gewölben eine Kappenschalung keineswegs erübrigte. In Meißen führte man die Gewölbe in Backsteinen im Format von etwa 28 x 13 x $9 \mathrm{~cm}$ ein Stein dick aus und sehr steil hinauf in den Dachraum. Ihr Zenit liegt knapp unterhalb der Zerrbalkenebene des Dachgerüstes. Waren die Gewölbekappen einmal aufgemauert, so hatte der Schlussstein kaum noch eine statische Funktion. Von der Vorstellung einer zweiteiligen Wölbstruk- 
tur aus einem tragenden Rippengerüst und darauf lastenden Gewölbekappen ausgehend, propagierte Eugène Violett-le-Duc (1814-1879) seine teilweise heute noch vertretene Idee ihrer Statik. Identische Rissbildungen bei Kreuzgrat- und Kreuzrippengewölben implizieren jedoch für beide Wölbtypen ein analoges Kräfteschema. Heute wissen wir, dass die Rippen nur unwesentlich zur Stabilität des Gewölbes beitragen ${ }^{26}$; mehr noch, dass sie den Gewölbeschub durch ihren eigenen vergrößern. Die im unsanierten Zustand gelegentlich feststellbaren parallelen Abrisse zeigen, dass die Rippen gerade so noch selbsttragend sind, sich aber jeder Stützwirkung des Gewölbes entziehen. Nicht die Rippen, die für die großen Lasten ohnehin viel zu schwach dimensioniert wären, tragen das Gewölbe, sondern bei ausreichender Verkeilung zwischen der Rippe und dem Kappenmauerwerk werden sie vor dem Herabstürzen bewahrt. Diese theoretisch hergeleitete Veranschaulichung bleibt eine starke Vereinfachung. In Wirklichkeit stellen Risse elastische Gelenke dar, so dass ein Bogentragwerk an mehreren Örtlichkeiten Risse bilden kann und trotzdem noch statisch bestimmt bzw. tragfähig ist. Nur die sichere Abschätzung der Gelenksteifigkeiten gestaltet sich so schwer, dass meist auf die vereinfachte Anschauung zurückgegriffen wird.

Die moderne Baustatik geht davon aus, dass die Kreuzgewölbe als Schalentragwerk wirken, die Rippen jedoch als standfeste Bögen angesehen werden, die nicht oder nur geringfügig einwirken. ${ }^{27}$ Wussten dies aber die alten Baumeister? Werner Müller äußerte sich skeptisch dazu. Für ihn scheint es keineswegs ausgeschlossen, dass die damaligen Meister „in der Rippe ein stabilisierendes Element gesehen haben“, dass sie aber gegen 1300 aus Erfahrung wussten, dass dem nicht so ist. ${ }^{28}$ Für die Stabilisierung der Rippen und der Kappen im unteren Drittel, dem Bereich der Gewölbeanfänger, half die Auffüllung der Räume zwischen Gewölbe und Sargmauer mit einem Mörtel-Schutt-Gemisch. Diese Konstruktion baute nicht nur den notwendigen Gegendruck gegen das Bersten der Gewölbebögen auf; das hohe Eigengewicht diente außerdem dazu, die Gewölbedrucklinie der idealen Senkrechten anzunähern. Dadurch kam der Meißner Baumeister mit der relativ geringen Bautiefe von lediglich 2,26 Meter im oberen Teil der $86 \mathrm{~cm}$,dicken“, lamellenartigen Strebepfeiler aus, die sich über drei Abstufungen bis auf 3,58 Meter nach unten hin vergrößerten. Diese radial angeordneten, extrem schlanken Mauerwerksscheiben ruhten auf den analog dimensionierten Substruktionen.

Alle diese konstruktiven Bauvorgaben beruhten offenbar auf der Intuition des Baumeisters, vor allem aber auf Erfahrung, die man nur auf Reisen sammeln konnte. Heute kann man über die Gleichgewichtsbeziehungen am Dreigelenkbogen die Größe der von einem Gewölbe verursachten Horizontalkomponente am Widerlager mit der Beziehung $\mathrm{H}=\left(\mathrm{g} \mathrm{x} \mathrm{l}^{2}\right):$ h beschreiben. Die Baumeister

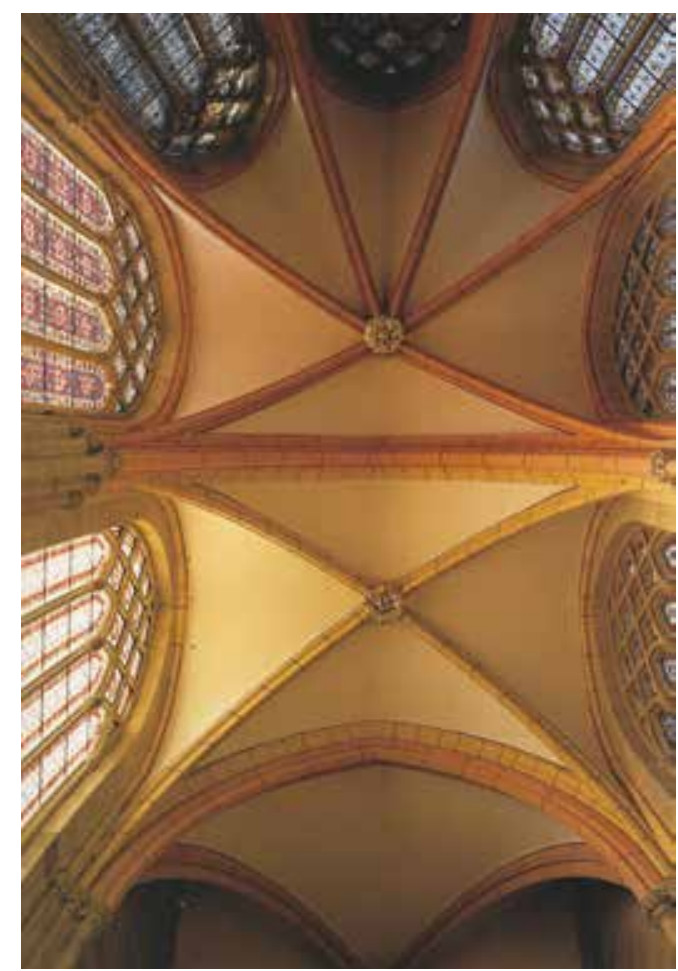

Blick in das Chorgewölbe des Meißner Domchors

๑ Hochstift Meißen

26 Norbert Nussbaum/Sabine Lepsky: Das gotische Gewölbe. Eine Geschichte seiner Form und Konstruktion. München 1999.

27 Zuletzt Rainer Barthel: Tragverhalten und Berechnung gemauerter Kreuzgewölbe. In: Fritz Wenzel (Hrsg.): Erhalten historischer Bauwerke. Baugefüge, Konstruktionen, Werkstoffe. Jahrbuch 1992. Berlin 1994.

28 Zitiert nach Werner Müller: Grundlagen gotischer Bautechnik. München 1990.

des Mittelalters mussten auch ohne statische Theorie, die es noch nicht gab, bei ihren Planungen auskommen. Sie taten aber alles nur Erdenkliche (und Richtige), um die Kraftgröße $\mathrm{H}$ zu minimieren: Sie verringerten das Eigengewicht $(\mathrm{g})$ durch
Blick in den Hohen Chor des Meißner Doms

Foto: Matthias Rutkowski

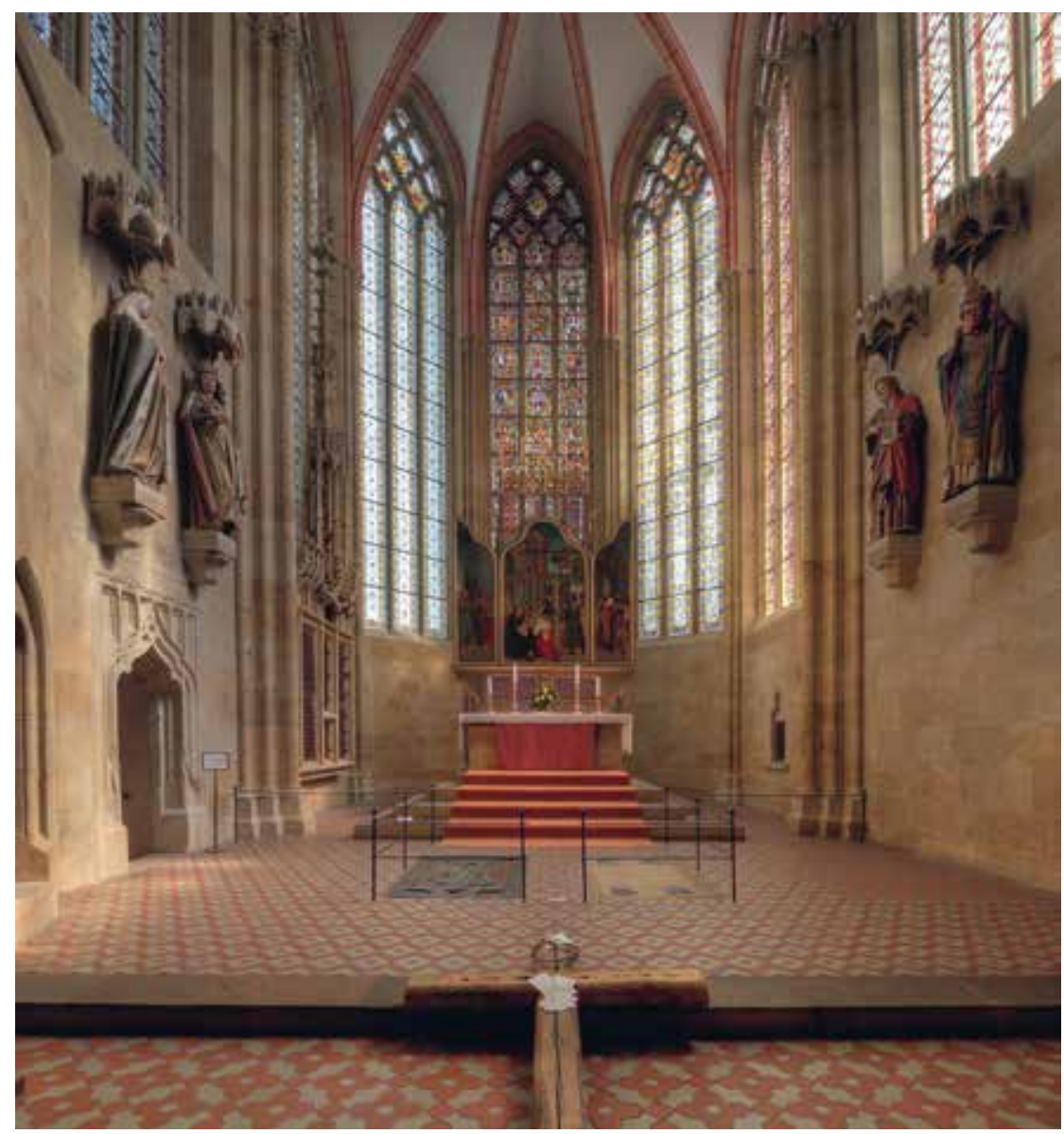


29 Ernst Gotthelf Gersdorf (Hrsg.): Urkundenbuch des Hochstifts Meißen. Bd. 1. (Codex diplomaticus Saxoniae regiae II, 1). Leipzig 1864, Nr. 145-146.

30 Matthias Donath: Die Grabmonumente im Dom zu Meißen. Leipzig 2004, S. 216.

31 CDS II, 1 (wie Anm. 29), Nr. 213. die Reduzierung der Gewölbedicke, sie erhöhten das Bogenstichmaß (h) und - das ist vor allem bemerkenswert - sie verringerten den quadratisch anzusetzenden Koeffizienten der Stützweite (1) durch die gezielte Anordnung auskragender Schichten. Was sie nicht ahnten war, dass sich dadurch die Vertikalkräfte wegen der fehlenden kraftschlüssigen Verbindung zwischen den beiden Steinschalen der Wände auf die innere Wandschale konzentrierten. Dabei wurden aber in vielen Fällen die Grenzen der vom Wandquerschnitt aufzunehmenden Querzugspannungen erreicht oder sogar überschritten - so auch in Meißen.

Der Schlussstein des Vierungsgewölbes wurde als gewaltiger Schlundring mit einer Öffnungsweite von etwa einem Meter Durchmesser ausgeführt. Darüber stand der Baukran - wahrscheinlich eine einfache Haspel - mit dem man das Baumaterial nach oben hieven konnte. Schlusssteine waren aber nicht nur konstruktiv wichtig; ihnen wurde im Mittelalter auch gern eine hohe symbolische Bedeutung zuteil. Indem man den Schlussstein als „Eckstein“ verstand, „der die Wände zusammenführt“, konnte er zum Symbol Christi werden. Damit war klar, weshalb gerade für die Meißner Schlusssteine alle Sorgfalt, Phantasie und Reichtümer aufgewendet worden sind. An den Rippen finden wir auch das Steinmetzzeichen des Dorsales und Lettners, das „T“, wieder: wahrscheinlich war dieser Bildhauer auch der Wölbmeister und Schöpfer der Schlusssteine; insgesamt kommt es im Abschnitt Ib 13 mal vor; es war auch in Naumburg und der Templerkapelle von Iben zu finden. Erst dann konnte mit dem inneren Auszieren, dem Verputz der Gewölbe, der Glasmalerei, den Vergoldungen und Farbfassungen der Bauabschnitt Ib abgeschlossen werden. Das Aufgehende umfasst für Ib:

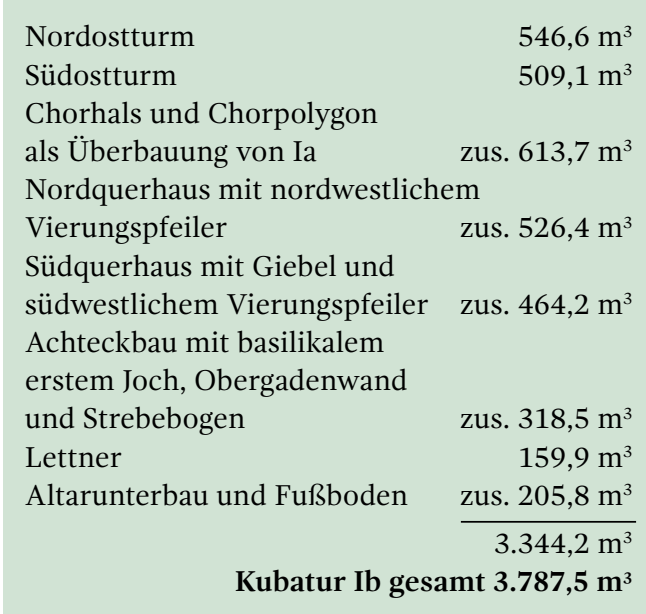

Für die Auffüllung des Mauerwerkskerns wurden ca. 600 Kubikmeter Bruchsteine und Hausteinabfälle benötigt. Bei dieser Menge konnte man die gesamten Abbruchmassen des romanischen Doms mit im gotischen Schalenmauerwerk verbauen. Im Zusammenhang mit der Einwölbung des Baus mussten weitere 100,4 Kubikmeter profilierte Gewölberippen, Jochbögen und reich verzierte
Schlusssteine über Bogenschablonen aufgerissen, gehauen und versetzt werden. Allein für das einzigartige Couronnement der Fenster und der Fensterpfosten wurden weitere 35 Kubikmeter Sandstein bearbeitet; die Verglasungsfläche beträgt ca. 282 Quadratmeter. Die Kubatur der Gewölbe umfasst 275 Kubikmeter; um sie zu bauen, mussten ca. 92.000 Backsteine vermauert werden. Für den Abbund der Dächer mussten 95 Kubikmeter Holz, wohl Weißtanne aus den Wäldern der fabrica bei Döbeln, gewonnen werden. Die Ziegelei musste neben den Backsteinen für die Gewölbe weitere noch für die Dacheindeckung mit Tonziegeln benötigte 52.500 Dachziegel brennen und liefern. Geht man davon aus, dass es in dieser Bauphase zu keinerlei Unterbrechung oder Verzögerung wegen Geldmangels o.ä. gab, ist von weiteren $4 \frac{1}{1} 2$ bis 5 Jahren Bauzeit auszugehen; davon entfallen mindestens drei Jahre für das Herstellen des Aufgehenden.

\section{Zusammenfassung}

Für den Bau der Ostteile des Doms als funktionsfähige Einheit ist eine unglaublich kurze Bauzeit von acht, höchstens aber zehn Jahren anzunehmen - eine Folge der Bauorganisation mit einem arbeitsteiligen ausgefeilten, technologisch hochentwickelten Verfahren des kreuzgewölbten Gliederbaus. Geht man davon aus, dass ein Teil der Arbeiter nach Abschluss der Arbeiten am Naumburger Westchor 1249 nach Meißen weiter zog - wie es an Hand der vergleichbaren Steinmetzzeichengruppen schlüssig nachgewiesen werden konnte - so wäre für Meißen ein Baubeginn um 1250, spätestens 1251 die logische Konsequenz. Hier eine zeitliche Zäsur zu setzen wäre unlogisch und auch nicht zu begründen. Das Jahr 1250 korreliert mit einer von Papst Innozenz IV. ausgestellten Urkunde, der denjenigen Gläubigen 40 Tage Ablass gewährte, die den Meißner Dom an den Festtagen der Kirchenpatrone besuchten. ${ }^{29}$. Bei der über die Baumassen ermittelten Bauzeit könnten die Ostteile also bereits 1260 fertiggestellt worden sein; als spätester Zeitpunkt wäre 1266 anzunehmen. Aus dieser Zeit stammt die Deckplatte des Sarkophags von Bischof Albert II. (1258-1266), der am 24. Juli 1266 verstorben ist und vor dem Kreuzaltar, also in der Vierung westlich des Lettners, am 1. August 1266 bestattet wurde $^{30}$. Da kaum anzunehmen ist, dass man den Bischof in einer Baustelle beigesetzt hat, ist davon auszugehen, dass der Dombau des Teilbauabschnittes Ib zu diesem Zeitpunkt bereits abgeschlossen sein musste. Deshalb scheidet die Annahme für einen Baubeginn „nicht vor 1260“ vollkommen aus. Insgesamt war der Bau der Domkirche jedoch noch nicht beendet. Darauf beziehen sich dann auch die späteren Urkunden, wie die vom 25. Januar 1271, in welcher der Bischof von Minden denen einen 40-tägigen Ablass erteilte, die der Fabrika milde Gaben zukommen lassen und die zur Vollendung des unvollendeten neuen Domes, der hohe Kosten verbraucht, beitrugen („opere novo tam sumptuoso viderimus inchaotam“). ${ }^{31}$ 